\title{
Cardiorespiratory considerations for return-to-play in elite athletes after COVID-19 infection: a practical guide for sport and exercise medicine physicians
}

\author{
Mathew G Wilson (D) 1,2 James H Hull (D) , 1,3,4 John Rogers, ${ }^{5,6,7}$ Noel Pollock, 1,8 \\ Miranda Dodd, ${ }^{2}$ Jemma Haines (D) , 5,6,9 Sally Harris, ${ }^{5,7}$ Mike Loosemore (D) , 1,4 \\ Aneil Malhotra (D) , $5,6,10$ Guido Pieles (1) ,"11 Anand Shah (1) , 3,12 Lesley Taylor, \\ Aashish Vyas, ${ }^{5,6,13}$ Fares S Haddad, ${ }^{1,2,14}$ Sanjay Sharma (i) ${ }^{15}$
}

For numbered affiliations see end of article.

\section{Correspondence to}

Professor Mathew G Wilson, Institute of Sport Exercise and Health (ISEH), University College London, London W1T 7HA, UK: mathew.wilson@hcahealthcare. co.uk

Accepted 11 August 2020 Published Online First 2 September 2020

\section{Linked}

- http://dx.doi.org/10.1136/ bjsports-2020-103259

Check for updates

(C) Author(s) (or their employer(s)) 2020. No commercial re-use. See rights and permissions. Published by BMJ.

To cite: Wilson MG,

Hull JH, Rogers J.

et al. Br J Sports Med

2020;54:1157-1161.

\section{ABSTRACT}

SARS-CoV-2 is the causative virus responsible for the COVID-19 pandemic. This pandemic has necessitated that all professional and elite sport is either suspended postponed or cancelled altogether to minimise the risk of viral spread. As infection rates drop and quarantine restrictions are lifted, the question how athletes can safely resume competitive sport is being asked. Given the rapidly evolving knowledge base about the virus and changing governmental and public health recommendations, a precise answer to this question is fraught with complexity and nuance. Without robust data to inform policy, return-to-play (RTP) decisions are especially difficult for elite athletes on the suspicion that the COVID-19 virus could result in significant cardiorespiratory compromise in a minority of afflicted athletes. There are now consistent reports of athletes reporting persistent and residual symptoms many weeks to months after initial COVID-19 infection. These symptoms include cough, tachycardia and extreme fatigue. To support safe RTP, we provide sport and exercise medicine physicians with practical recommendations on how to exclude cardiorespiratory complications of COVID-19 in elite athletes who place high demand on their cardiorespiratory system. As new evidence emerges, guidance for a safe RTP should be updated.

\section{INTRODUCTION}

SARS-CoV-2 is the causative virus responsible for the COVID-19 pandemic. The COVID-19 pandemic has necessitated that all professional sport is either suspended, postponed or cancelled altogether to minimise the risk of viral spread. As infection rates drop and quarantine restrictions are lifted, questions are being asked of how athletes can safely resume competitive sport. Given the rapidly evolving knowledge base about the virus and changing governmental and public health recommendations, ${ }^{1}$ precise answers to this highly relevant question is fraught with complexity and nuance. ${ }^{2}$ Without robust data to inform policy, return-toplay (RTP) decisions are especially difficult for elite athletes on the suspicion that the COVID-19 virus could result in significant cardiorespiratory compromise in a minority of afflicted athletes. There are now consistent reports that COVID-19 positive athletes may present persistent and residual symptoms many weeks to months after initial infection, including cough, tachycardia and extreme fatigue.

To support safe RTP, we provide sport and exercise medicine physicians with practical recommendations on how to exclude cardiorespiratory complications of COVID-19 in elite athletes who place high demand on their cardiorespiratory system. Given our rapidly emerging understanding of COVID-19, these recommendations are subject to adjustments as new evidence becomes available.

\section{CARDIAC SYSTEM}

Data from China suggest that COVID-19 is associated with biochemical evidence of cardiac myocyte necrosis in almost one in five hospitalised patients and that patients with elevated serum cardiac troponin concentrations above the 99th percentile are more likely to require mechanical ventilation and/or die than those who do not have raised troponins. $^{3-6}$ The precise mechanism for myocyte necrosis is unclear; however, current observations from the kinetics of the magnitude and duration of the elevation in troponin concentration suggest the most common cause reflects the systemic inflammatory response or cytokine storm associated with the infection. Other mechanisms include profound hypoxaemia from the respiratory illness, right ventricular strain due to hypoxaemic pulmonary vasocontriction or thromboembolic complications, inflammatory atherosclerotic plaque rupture with distal embolisation or viral myocarditis. It is important to emphasise that such complications were apparent in patients considered ill enough to warrant hospitalisation; therefore, the prevalence of myocardial injury or raised troponin in athletes with asymptomatic infections or a relatively mild illness is unknown but likely to be low.

It is also recognised that the SARS-CoV-2 virus binds to ACE 2 (ACE2) in the upper airways and lungs after the protein spike on the virus is activated by transmembrane protease serine $2 .^{7}$ The ACE2 receptor is a membrane protein found in abundance in the lungs and serves many protective pathways. The myocardium also contains a high concentration of ACE2 receptors, activation of which may facilitate direct toxic effects within the myocardium or offset the effects of the renin angiotensin aldosterone system. ${ }^{7}$ A rare proportion of patients who 
have been diagnosed with COVID-19 infection have presented with chest pain and palpitations rather than symptoms due to respiratory tract involvement. ${ }^{3}$

For athletes suffering with flu-like symptoms, myocarditis has always been a potential complication of viral syndromes. Although the diagnosis is reliant on histological confirmation or the demonstration of myocardial oedema or late gadolinium enhancement (LGE) on cardiac MRI, emerging case reports appear to implicate myocarditis as an additional cause of cardiac damage from COVID-19. ${ }^{8}$ Key symptoms include chest pain that may be made worse by deep inspiration, increasing breathlessness and palpitations. Physical signs may be few; however, particular attention should be given to tachycardia, added heart sounds, bibasal crackles or signs of a pleural effusion. While serum biomarkers of myocardial damage are commonly elevated in the acute stages, it important to note that raised troponins do not equate to a diagnosis of myocarditis and may be secondary to intensive physical training. ECG manifestations may include non-specific signs such as ST-segment elevation or depression, T-wave inversion and/or ventricular arrhythmias. It is noteworthy that repolarisations anomalies are also common to the electrical adaptations to athletic training. Cardiac imaging techniques might show global or regional wall motion abnormalities along with variable degrees of pericardial effusion. Myocardial oedema might be identified in early stages with cardiac MRI, while LGE can be present early in its evolution and remain as a sequela. ${ }^{10}$ From available data thus far, it appears there is right ventricular (RV) impairment over left ventricular (LV) involvement in previously hospitalised patients with COVID-19 infection. ${ }^{11}$ The incidence of myocarditis associated arrhythmias is unknown, and while COVID-19 infection led to a $\geq 50 \%$ increase in out-of-hospital cardiac arrest in the general population, ${ }^{12}$ the data are descriptive and do not necessarily suggest an increased risk of sudden cardiac arrest or arrhythmias in otherwise 'healthy' COVID-19 positive individuals. Animal studies have, however, shown that exercising with myocarditis can increase viral replication and inflammation within the heart, resulting in permanent damage or occasionally sudden death. ${ }^{13}$ Indeed, myocarditis accounted for $20 \%$ of all non-traumatic sudden death among a cohort of military recruits. ${ }^{14}$

\section{CARDIAC RTP PATHWAY}

To prevent sudden death in athletic populations, most professional and elite sport federations have an existing preparticipation cardiovascular evaluation protocol, ${ }^{15}$ often using 12-lead ECG \pm echocardiography (ECHO). While this document does not stray far from established recommendations, we propose minor pathway modifications based on reasonable clinical indications (figure 1).

1. For athletes who have not presented with signs or symptoms of COVID-19 during the pandemic, we recommend that no additional cardiac investigations are required before RTP.

2. Athletes with mild to moderate COVID-19 symptoms (eg, who managed their condition at home and have recovered) pose more of a challenge. Few will have had testing with confirmed COVID-19 infection and most will simply suspect they had the infection. For those that have fully recovered without ongoing cardiovascular symptoms (symptom free at rest for 7 days and no sooner than day 10 from the onset of symptoms), we recommend a thorough clinical assessment with a medical history and physical examination. It is essential that physicians managing potentially affected athletes are equipped with personal protective equipment and pay par-

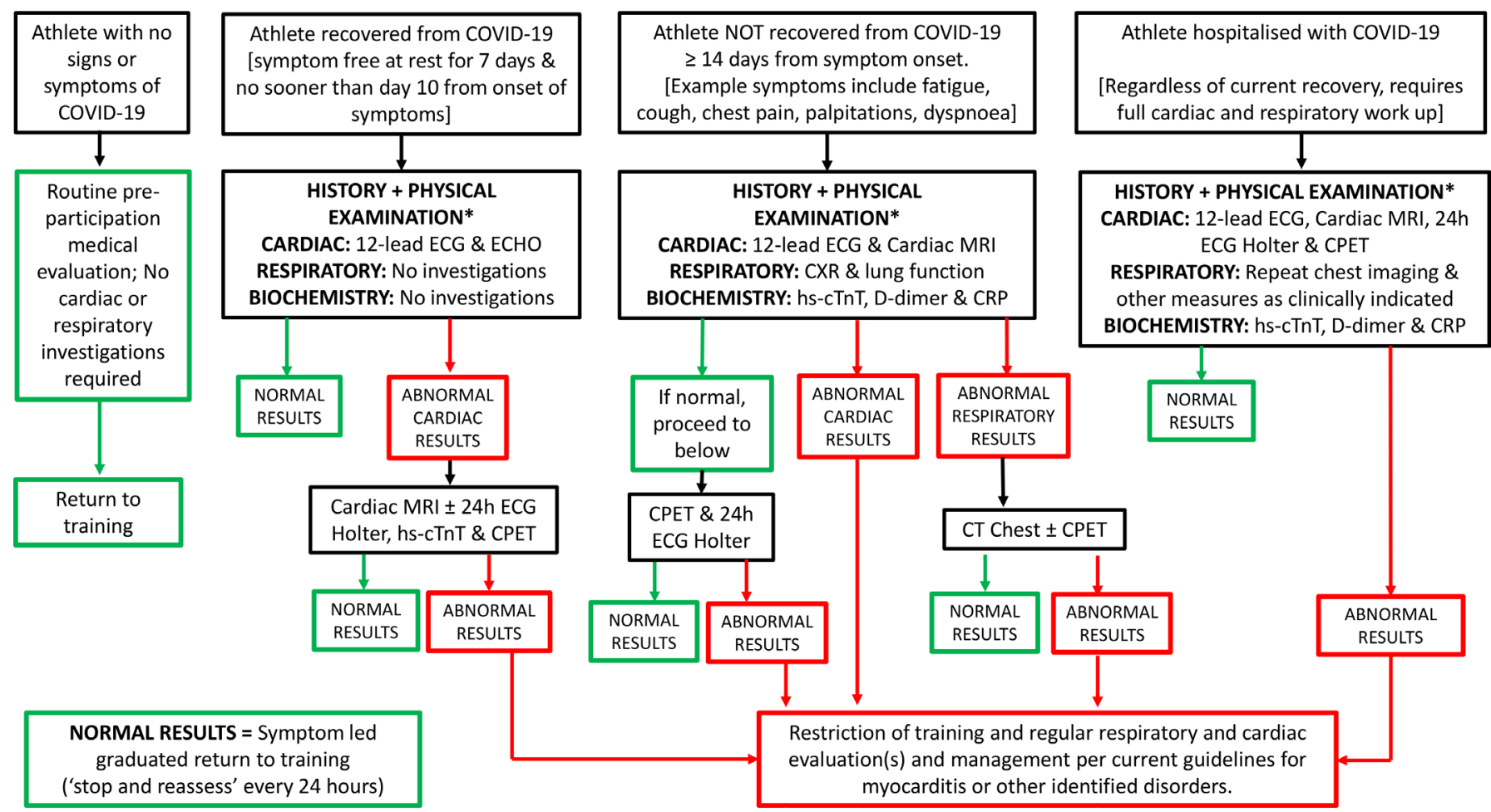

Figure 1 RTP pathway in those elite athletes confirmed (or suspected) COVID-19 positive. *History and physical examination should also consider other organ systems where COVID-19 can have pathological consequences such as neurological, gastrointestinal and dermatological. CPET, cardiopulmonary exercise test; CRP, C reactive protein; CXR, chest X-ray; ECG, electrocardiogram; ECHO, echocardiography; hs-cTnT; high-sensitivity cardiac troponin $\mathrm{T}$; MRI, magnetic resonance imaging; RTP, return to play. 
ticular attention to cleansing the stethoscope with detergent wipes after use. The preliminary assessment should focus on the presence of chest pain, dyspnoea, palpitation, exertional dizziness, syncope, tachycardia, added heart sounds, basal crepitations or reduced air entry. We also recommend a minimum of additional cardiac testing including 12-lead ECG and ECHO before RTP. If abnormalities are observed, a cardiac MRI should be performed to exclude myocarditis. Other secondary investigations may include cardiopulmonary exercise testing and 24-hour Holter ECG.

3. Athletes with persistent COVID-19 symptoms may take longer than 14 days to recover. For this group, we recommend a thorough history and physical examination, 12-lead ECG and cardiac MRI to check specifically for myocarditis. If the MRI is normal, then proceed to cardiopulmonary exercise testing and 24-hour Holter ECG. This stepwise approach is to ensure athletes who are still ill are not made to exercise maximally until initial investigations have been completed.

4. Athletes who experienced COVID-19 symptoms severe enough to require admission to hospital, we recommend a full cardiac work-up including; 12-lead ECG, cardiac MRI, cardiopulmonary exercise testing and 24-hour Holter ECG.

\section{Role of serum cardiac troponin}

The panel recommends that measurement of serum cardiac troponin should be confined to athletes with ongoing symptoms compatible with myocarditis and those where imaging studies reveal impaired myocardial function. In such circumstance, the athlete should be rested for at least 48 hours before investigation as intensive exercise itself may be associated with a transient increase in serum cardiac troponin concentration. Routine troponin assessment in all athletes who may have had COVID-19 infection is not recommended because there are no validated cut-off values for cardiac involvement in COVID-19 and baseline troponin levels during normal health will not be available for most athletes.

\section{Annual medical timings}

Some elite clubs may choose to perform 12-lead ECG and/or ECHO through expediting the usual pre-season annual medical evaluation. If ECG is performed, it would be desirable to compare with previous ECGs as some repolarisation changes detected in myocarditis overlap with those observed in athletic training.

\section{RESPIRATORY SYSTEM}

It is anticipated that the majority of athletes with mild to moderate COVID-19 infection will fully recover with little need for enhanced respiratory assessment. Many athletes are, however, reporting a persistent cough and dyspnoea following infection, especially in the context of vigorous exercise. It is anticipated that in the majority of these cases, symptoms will fully resolve in the 4-week period following infection and that recovery should be progressive. ${ }^{16}$ Accordingly, any deterioration or deviation from a pattern of daily improvement and recovery or the development of new symptoms (eg, new productive cough, chest pain or worsening dyspnoea) should prompt immediate reappraisal with a temporary cessation of the RTP programme, until clinical work-up has been completed. This evaluation should begin with a chest X-ray, D-Dimer and pulmonary function testing to check for new or worsening pneumonia, pulmonary embolism or postinflammatory bronchoconstriction. Due to the potential overlap of cardiovascular and pulmonary symptoms, athletes with persistent cardiorespiratory symptoms should also undergo a comprehensive cardiac evaluation as previously outlined.

In individuals hospitalised with COVID-19 related respiratory illness, the presence of radiographic pneumonic change will be ubiquitous. Emerging data also indicate that moderate to severe disease is associated with a high prevalence of thrombotic events and as such it is likely that the potential sequela of COVID-19 pneumonic illness predominantly impacts the pulmonaryvascular interface. ${ }^{17}$ This has implications for selection of the best tests to assess any post-COVID-19 respiratory limitation and also highlights the importance of sports and exercise medicine clinicians being aware that any post-COVID-19 breathlessness and/or chest pain may be arising due to serious pulmonary vascular pathology such as pulmonary embolism. Overall, we recommend that any athletic individual that has been hospitalised with a radiologically confirmed COVID-19 pneumonia and breathlessness undergoes specialist respiratory review prior to RTP, and this process is likely to involve the need for: (1) planned repeat imaging; (2) baseline physiological measures (including consideration of gas transfer measurement \pm lung volumes); and (3) the possible need for cardiopulmonary exercise testing with measurement of oxygen saturation in selected cases with ongoing dyspnoea on exertion.

Chronic post-COVID-19 respiratory symptoms may include: breathlessness on exertion, new onset or persistence of a postCOVID cough and wheeze and/or chest tightness that may or may not be related to exertion. In athletic populations, there is a high prevalence of background airways disease, with studies consistently showing that approximately one in four endurance athletes has evidence of airway dysfunction (eg, asthma \pm exercise-induced bronchoconstriction), and in many cases, this remains undetected and is only identified at screening assessment. ${ }^{18} 19$ Thus, a contribution from underdiagnosed or undertreated airways disease that has been exacerbated by COVID-19 infection should not be overlooked. We speculate at this point that COVID-19 infection could provoke new onset asthma-like symptoms (which may also include exercise-induced laryngeal obstruction). Where applicable, it is vital that athletes are encouraged to strongly adhere to their routinely prescribed respiratory medication and clinicians should review inhaler technique and appropriate use of spacing devices. In cases of suspected airways disease, assessment with spirometry \pm bronchodilator challenge \pm bronchoprovocation testing and assessment of airway inflammation (eg, with exhaled nitric oxide (FeNO)) is recommended. ${ }^{20}$ In order to optimise respiratory care, it is also important that allied respiratory issues are considered, for example, management of hay fever and allergies and any undertreated reflux. ${ }^{21}$ If under-recognised and undertreated, then these factors may also contribute to ongoing exercise cough. ${ }^{22}$

In some cases, COVID-19 may cause chest restriction (eg, from pain or local areas of pulmonary collapse), present during the height of the pneumonia phase, which subsequently precipitate disordered breathing patterns. This may respond to the timely initiation of respiratory physiotherapy intervention. Symptoms of early fatigability and reduced exercise performance may also be features of an increased work of breathing due to underlying respiratory disease and should also prompt a respiratory investigation.

At the current time, it is difficult to fully know the long-term implications of post-COVID-19 lung disease from severe spectrum disease, and the evidence base that does exist is based on a small sample size or extrapolated from previous experience of SAR-CoV-1 and Middle East respiratory syndrome coronavirus. 
Severe COVID-19 related respiratory disease is associated with damage to the pulmonary airspaces, interstitium and pulmonary vascular interface. Preliminary data indicate that the most sensitive pulmonary function test in this context is the measurement of gas transfer. ${ }^{23}$ Venous thrombosis in the lungs with or without the development of secondary pulmonary hypertension may occur. ${ }^{24} 25$ Case study reports in individuals with acute respiratory distress syndrome during their acute illness leading to pulmonary fibrosis and exertional breathlessness are present, though the prevalence of such presentations and implications for athletes with more mild disease is unknown.

\section{RESPIRATORY RTP PATHWAY}

1. For athletes without symptoms or signs of COVID-19 during the pandemic, no formal respiratory testing is required (figure 1). Those athletes with documented underlying respiratory issues, such as asthma, should continue treatment and have management optimised accordingly.

2. Athletes with mild to moderate COVID-19 symptoms who managed their condition at home but are now fully recovered (symptom free at rest for 7 days and no sooner than day 10 from the onset of symptoms), no formal respiratory testing is required. Any recovery process should be progressive and thus, any deviation should result in a 'stop and reassess' situation. Those athletes with documented underlying respiratory issues, such as asthma, should continue treatment and have management optimised accordingly.

3. In those athletes who report COVID-19 related respiratory symptoms that are persistent and taking longer than 14 days to recover, we recommend a thorough assessment to exclude the presence of thromboembolic events, ongoing intrapulmonary pathology or cardiac injury. This assessment should consider a chest X-ray, ECG, biomarkers for inflammation, myocyte necrosis or thromboembolic disease (including C reactive protein, hs-cTnT and D-dimer if not arranged in the past) and lung function. It is also logical to discuss case management with a respiratory physician. In cases with a significant suspicion of thromboembolic or intrapulmonary abnormalities, we recommend a CT thorax with due consideration for the correct imaging protocol to identify postCOVID-19 changes plus pulmonary vasculature pathology (eg, thromboembolism). If the cause of the breathlessness remains elusive, then proceed to a cardiopulmonary exercise test with $\mathrm{O}_{2}$ saturation but ideally with blood gas monitoring. If cases of desaturation during exercise with a normal CT, consider a ventilation-perfusion scan for possible microemboli. If spirometry demonstrates evidence of obstructive airways disease, this should prompt consideration for a new diagnosis of asthma/postinfective bronchial hyper-reactivity and the assessment of bronchodilator reversibility and the presence of airways inflammation (eg, with FeNO), prior to planning treatment.

4. For athletes who have experienced respiratory symptoms severe enough to require hospital admission, we recommend a full respiratory review prior to RTP. In this scenario, it is likely that chest imaging and other measures (eg, troponin and D-dimer) will have already been undertaken, so management will be individualised based on prior findings and the recovery course.

\section{OTHER RTP CONSIDERATIONS}

While a pragmatic approach is recommended, we recognise our proposed RTP pathway should be interpreted and modified on an individual basis (figure 1). It is important to consider that: (1) our RTP pathway is intended for elite athletes (eg, premier league football, professional rugby, potential Olympiads and so on) who are currently being required to return to train and compete, (2) a standard cardiac evaluation typically performed during preseason may be sufficient in the vast majority of athletes to allow RTP, (3) most athletes will have previous preparticipation medical evaluations that can be used for comparison, (4) athletes with significant and prolonged symptoms or those that were hospitalised from COVID-19 require a complete cardiac and respiratory workup even if now fully recovered and finally (5) in athletes that are/were infected with confirmed or suspected COVID-19, any graded RTP decision starts when athletes are symptom free at rest for 7 days and no sooner than 10 days from the onset of symptoms.

While the primary COVID-19 concerns have focused on the cardiorespiratory system, COVID-19 can have pathological consequences on other organ systems that may influence RTP decision making in athletes. When undertaking a detailed history and physical examination, consideration should also be given to the neurological, gastrointestinal and dermatological systems. ${ }^{26}$ Where recommendations are provided, especially for aerosolgenerating procedures (ie, spirometry and pulmonary function tests), medical facilities and clinicians should adhere to strict personal protective equipment policies and procedures.

Finally, physical and psychological deconditioning, mental health and underperformance issues may also be present as athletes emerge from quarantine restrictions. Some athletes have already raised concern in the media with respect to health anxieties related to themselves or their families, particularly for black, Asian and ethnic minorities, where they are at higher risk of death from COVID-19. ${ }^{27}$ For athletes recovering from COVID-19, it is also important to consider the psychological impacts of a prolonged recovery. It is well known that psychological factors effect morbidity outcomes ${ }^{28}$ and athletes are no different. The English Institute of Sport has published useful advice on the psychosocial considerations of phased return for athletes (health adjustment and transition). ${ }^{29}$

\section{CONCLUSION}

In conclusion, we provide practical recommendations on how to medically evaluate the cardiorespiratory system of the athletes to safely return to intensive training and competitive sport. The potential for cardiorespiratory complications from COVID-19 requires a careful assessment based on the clinical and symptom course and severity of illness. We appreciate a pragmatic approach must be taken and suggest our recommendations are interpreted on an individual basis. Clinical assessment, RTP planning and review (progress) are circular in nature. This considers the dynamic nature of RTP with continual focus on the athlete's progress and assessment for new symptoms.

\footnotetext{
Author affiliations

${ }^{1}$ Institute for Sport Exercise and Health (ISEH), University College Hospital London, London, UK

${ }^{2}$ The Princess Grace Hospital (HCA Healthcare UK), London, United Kingdom ${ }^{3}$ Department of Respiratory Medicine, Royal Brompton Hospital, London, UK ${ }^{4}$ English Institute of Sport, London, United Kingdom

${ }^{5}$ Manchester Institute of Health \& Performance (MHIP), Manchester, United Kingdom ${ }^{6}$ Manchester University NHS Foundation Trust, Manchester, United Kingdom

${ }^{7}$ The Wilmslow Hospital (HCAHealthcareUK), Wilmslow, United Kingdom ${ }^{8}$ British Athletics, London, United Kingdom

${ }^{9} \mathrm{NIHR}$ Manchester BRC and University of Manchester, Manchester, United Kingdom

${ }^{10}$ Department of Cardiovascular Science, University of Manchester, Manchester, United Kingdom

${ }^{11}$ Cardiovascular Biomedical Research Centre, Bristol Heart Institute, Bristol, UK
} 
${ }^{12}$ Department of Infectious Diseases Epidemiology, Imperial College London, London, United Kingdom

${ }^{13}$ Lancashire Teaching Hospitals Trust, Lancashire, United Kingdom

${ }^{14}$ Department of Trauma and Orthopaedics, University College London Hospitals NHS Foundation Trust, London, London, United Kingdom

${ }^{15}$ St. George's University Hospitals NHS Foundation Trust, London, United Kingdom

Twitter Mathew G Wilson @Prof_MatWilson, James H Hull @Breathe_to_win, John Rogers@jpmrogers, Noel Pollock@DrNoelPollock, Jemma Haines @jemhaines, Mike Loosemore @doctorloosemore, Aneil Malhotra @DrAneilMalhotra, Aashish Vyas @EB_clinic, Fares S Haddad @bjjeditor and Sanjay Sharma @SSharmacardio

Contributors MGW manuscript conception. MGW, JHH, JR, NP, FH and SS wrote first draft. All authors edited and approved final document.

Funding No public, commercial or not-for-profit funding was provided for this document.

Competing interests None declared.

Patient consent for publication Not required.

Provenance and peer review Not commissioned; externally peer reviewed.

Data availability statement There are no data in this work.

This article is made freely available for use in accordance with BMJ's website terms and conditions for the duration of the covid-19 pandemic or until otherwise determined by BMJ. You may use, download and print the article for any lawful, non-commercial purpose (including text and data mining) provided that all copyright notices and trade marks are retained.

\section{ORCID iDs}

Mathew G Wilson http://orcid.org/0000-0002-6317-0168

James H Hull http://orcid.org/0000-0003-4697-1526

Jemma Haines http://orcid.org/0000-0003-3813-041X

Mike Loosemore http://orcid.org/0000-0002-4855-0744

Aneil Malhotra http://orcid.org/0000-0002-8670-3764

Guido Pieles http://orcid.org/0000-0003-1203-688X

Anand Shah http://orcid.org/0000-0001-5257-520X

Sanjay Sharma http://orcid.org/0000-0002-3630-6138

\section{REFERENCES}

1 Elite sport return to training guidance: step one. Available: https://www.gov.uk/ government/publications/coronavirus-covid-19-guidance-on-phased-return-of-sportand-recreation/elite-sport-return-to-training-guidance-step-one--2

2 Hull JH, Loosemore M, Schwellnus M. Respiratory health in athletes: facing the COVID-19 challenge. Lancet Respir Med 2020;8:557-8.

3 Huang C, Wang Y, Li X, et al. Clinical features of patients infected with 2019 novel coronavirus in Wuhan, China. The Lancet 2020;395:497-506.

4 Shi S, Qin M, Shen B, et al. Association of cardiac injury with mortality in hospitalized patients with COVID-19 in Wuhan, China. JAMA Cardiol 2020;5:802-10.

5 Guo T, Fan Y, Chen M, et al. Cardiovascular implications of fatal outcomes of patients with coronavirus disease 2019 (COVID-19). JAMA Cardiol 2020;5:811-8.

6 Zhou F, Yu T, Du R, et al. Clinical course and risk factors for mortality of adult inpatients with COVID-19 in Wuhan, China: a retrospective cohort study. Lancet 2020;395:1054-62.

7 Turner AJ, Hiscox JA, Hooper NM. Ace2: from vasopeptidase to SARS virus receptor. Trends Pharmacol Sci 2004;25:291-4.
8 Sala S, Peretto G, Gramegna M, et al. Acute myocarditis presenting as a reverse Tako-Tsubo syndrome in a patient with SARS-CoV-2 respiratory infection. Eur Heart J 2020;41:1861-2.

9 Inciardi RM, Lupi L, Zaccone G, et al. Cardiac involvement in a patient with coronavirus disease 2019 (COVID-19). JAMA Cardiol 2020. doi:10.1001/ jamacardio.2020.1096. [Epub ahead of print: 27 Mar 2020].

10 Lewis AJM, Burrage MK, Ferreira VM. Cardiovascular magnetic resonance imaging for inflammatory heart diseases. Cardiovasc Diagn Ther 2020;10:598-609.

11 Huang L, Zhao P, Tang D, et al. Cardiac involvement in patients recovered from COVID-2019 identified using magnetic resonance imaging. JACC Cardiovasc Imaging 2020. doi:10.1016/j.jcmg.2020.05.004. [Epub ahead of print: 12 May 2020].

12 Baldi E, Sechi GM, Mare C, et al. Out-Of-Hospital cardiac arrest during the Covid-19 outbreak in Italy. N Eng/ J Med 2020;383:496-8.

13 Karjalainen J, Heikkilä J, Nieminen MS, et al. Etiology of mild acute infectious myocarditis. Relation to clinical features. Acta Med Scand 1983;213:65-73.

14 Eckart RE, Scoville SL, Campbell CL, et al. Sudden death in young adults: a 25-year review of autopsies in military recruits. Ann Intern Med 2004;141:829-34.

15 Mont L, Pelliccia A, Sharma S, et al. Pre-participation cardiovascular evaluation for athletic participants to prevent sudden death: position paper from the EHRA and the EACPR, branches of the ESC. endorsed by APHRS, Hrs, and SOLAECE. Eur J Prev Cardiol 2017;24:41-69.

16 How long does COVID-19 last? Available: https://covid.joinzoe.com/post/covid-longterm [Accessed 11 Jun 2020].

17 Bompard F, Monnier H, Saab I, et al. Pulmonary embolism in patients with COVID-19 pneumonia. Eur Respir J 2020;56:2001365.

18 Hull JH, Ansley L, Robson-Ansley P, et al. Managing respiratory problems in athletes. Clin Med 2012;12:351-6.

19 Ansley L, Kippelen P, Dickinson J, et al. Misdiagnosis of exercise-induced bronchoconstriction in professional soccer players. Allergy 2012;67:390-5.

20 Parsons JP, Hallstrand TS, Mastronarde JG, et al. An official American thoracic Society clinical practice guideline: exercise-induced bronchoconstriction. Am J Respir Crit Care Med 2013;187:1016-27.

21 Olin JT, Hull JH. Exercise and the total airway: a call to action. Immunol Allergy Clin North Am 2018;38:xv-xix.

22 Hull JH, Dickinson JW, Jackson AR. Cough in exercise and athletes. Pulm Pharmacol Ther 2017:47:49-55.

23 Mo X, Jian W, Su Z, et al. Abnormal pulmonary function in COVID-19 patients at time of hospital discharge. Eur Respir J 2020;55. doi:10.1183/13993003.01217-2020. [Epub ahead of print: 18 Jun 2020].

24 Cui S, Chen S, Li X, et al. Prevalence of venous thromboembolism in patients with severe novel coronavirus pneumonia. J Thromb Haemost 2020;18:1421-4.

25 Klok FA, Kruip MJHA, van der Meer NJM, et al. Incidence of thrombotic complications in critically ill ICU patients with COVID-19. Thromb Res 2020;191:145-7.

26 Gupta A, Madhavan MV, Sehgal K, et al. Extrapulmonary manifestations of COVID-19. Nat Med 2020;26:1017-32.

27 Collaborative TO, Williamson E, Walker AJ, et al. OpenSAFELY: factors associated with COVID-19-related Hospital death in the linked electronic health records of 17 million adult NHS patients. medRxiv 2020.

28 Meeting the psychological needs of people recovering from severe coronavirus. pdf. Available: https://www.bps.org.uk/sites/www.bps.org.uk/files/Policy/Policy\% 20-\%20Files/Meeting\%20the\%20psychological\%20needs\%20of\%20people\% 20recovering\%20from\%20severe\%20coronavirus.pdf [Accessed 14 May 2020].

29 CV19-Psycho-Social-Considerations-of-Phased-Return.pdf. Available: https://www. eis2win.co.uk/app/uploads/2020/06/CV19-Psycho-Social-Considerations-of-PhasedReturn.pdf [Accessed 12 Jun 2020]. 